\title{
Hydroxychloroquine- and Azithromycin-Induced Transient Left-Bundle Branch Block in a Patient with COVID-19
}

Authors:

Disclosure:

Disclaimer:

Received:

Accepted:

Keywords:

Citation:
*Layal Mansour, ${ }^{1}$ Soha Fakhreddine,${ }^{2}$ Michelle Saliba, ${ }^{2}$ Samer Kabbani'

1. Department of Cardiovascular Medicine, Rafic Hariri University Hospital, Beirut, Lebanon

2. Department of Infectious Diseases, Rafic Hariri University Hospital, Beirut, Lebanon

*Correspondence to layal.mansour@live.com

The authors have declared no conflicts of interest.

The authors confirm that written consent for submission and publication of this case report, including images and associated text, has been obtained from the patient in line with Committee on Publication Ethics (COPE) guidance.

11.10 .20

01.03 .21

Arrhythmia, azithromycin (AZT), COVID-19, hydroxychloroquine (HCQ), left-bundle branch block (LBBB), pandemic, QT interval, QT prolongation, Torsade de Pointes (TdP), transient.

EMJ Cardiol. 2021;9[1]:92-97.

\section{Abstract}

Background: COVID-19 has emerged and rapidly spread worldwide due to the high infectivity of the novel coronavirus. A new regimen consisting of a combination of hydroxychloroquine and azithromycin has been under evaluation for efficacy and side effects, especially cardiotoxicity.

Case summary: A 58-year-old man was admitted to the hospital for COVID-19 pneumonia. His initial ECG showed sinus tachycardia. He was started on combination therapy of azithromycin and hydroxychloroquine. After the second dose of hydroxychloroquine and initial dose of azithromycin, his ECG showed complete left-bundle branch block (LBBB). The treatment was stopped, and the patient had no cardiac symptoms. On Day 8 of admission, his repeat ECG showed an absence of LBBB.

Discussion: The cumulative dose of hydroxychloroquine observed in patients treated for malaria or systemic diseases is cardiotoxic, and few cases of LBBB, have been reported. It is, however, not known whether the use of azithromycin in association with a small dose of hydroxychloroquine induces transient LBBB.

\section{INTRODUCTION}

Since the emergence of the novel coronavirus (referred to as severe acute respiratory syndrome coronavirus 2 [SARS-Cov-2]) in December

2019 in Wuhan City, China, and up until now, several studies and trials have been conducted to manage infected patients. Over the last few weeks, the focus moved from fear to hope in the form of the recently approved regimen (the 
combination of hydroxychloroquine [HCQ] and azithromycin [AZT])'. This treatment, however, has its own limitations and cardiac adverse effects including arrhythmias, QT prolongation, and Torsade de Pointes (TdP). ${ }^{1}$

The authors report here the case of a male patient diagnosed with COVID-19 pneumonia, who was started on this regimen and developed transient complete left-bundle branch block (LBBB) as a consequence.

\section{TIMELINE}

Day O: A 58-year-old man was admitted with possible COVID-19 pneumonia.

Day 1: Real-time (RT)-PCR was positive. His baseline ECG showed sinus tachycardia. He was started late that day on $\mathrm{HCQ}$ and AZT.

Day 2: He received a second HCQ dose. Repeat ECG showed complete LBBB. Combination therapy was stopped. Daily ECG was performed and LBBB persisted.

Day 8: The ECG reverted spontaneously normal.

\section{CASE PRESENTATION}

A 58-year-old man, non-smoker, with a medical history significant for hypertension, who recently came back from Liberia to Lebanon, presented to hospital on $16^{\text {th }}$ April 2020 with a productive cough and pleuritic chest pain. He denied fever or dyspnoea. His symptoms started a couple of days following his return from travel. Upon presentation, he was anxious but clinically stable. His oxygen saturation was $98 \%$ on ambient air, his heart rate 95 beats per minute, blood pressure $180 / 90 \mathrm{mmHg}$, and had a temperature of 36.1 ${ }^{\circ} \mathrm{C}$. Chest auscultation was unremarkable; heart sounds were regular without murmurs. He had no peripheral oedema.

A nasopharyngeal swab for RT-PCR COVID-19 was sent the same day of presentation and he was admitted to the COVID-19 unit. Laboratory workup of admission, including a complete blood-cell count, blood urea nitrogen, creatinine, electrolytes, magnesium, liver function tests, and cardiac enzymes were unremarkable. His ECG on admission showed sinus tachycardia with occasional premature ventricular complexes. PR interval was 200 ms, QT 320 ms, QTc 356 ms, by Bazett Formula (Figure 1).

A CT scan of the chest without intravenous contrast administration showed a faint focal ground-glass patch in the posterior segment of the left lower lobe, which was related to early COVID-19 pneumonia. The heart was normal in size. There were no significant abnormalities otherwise.

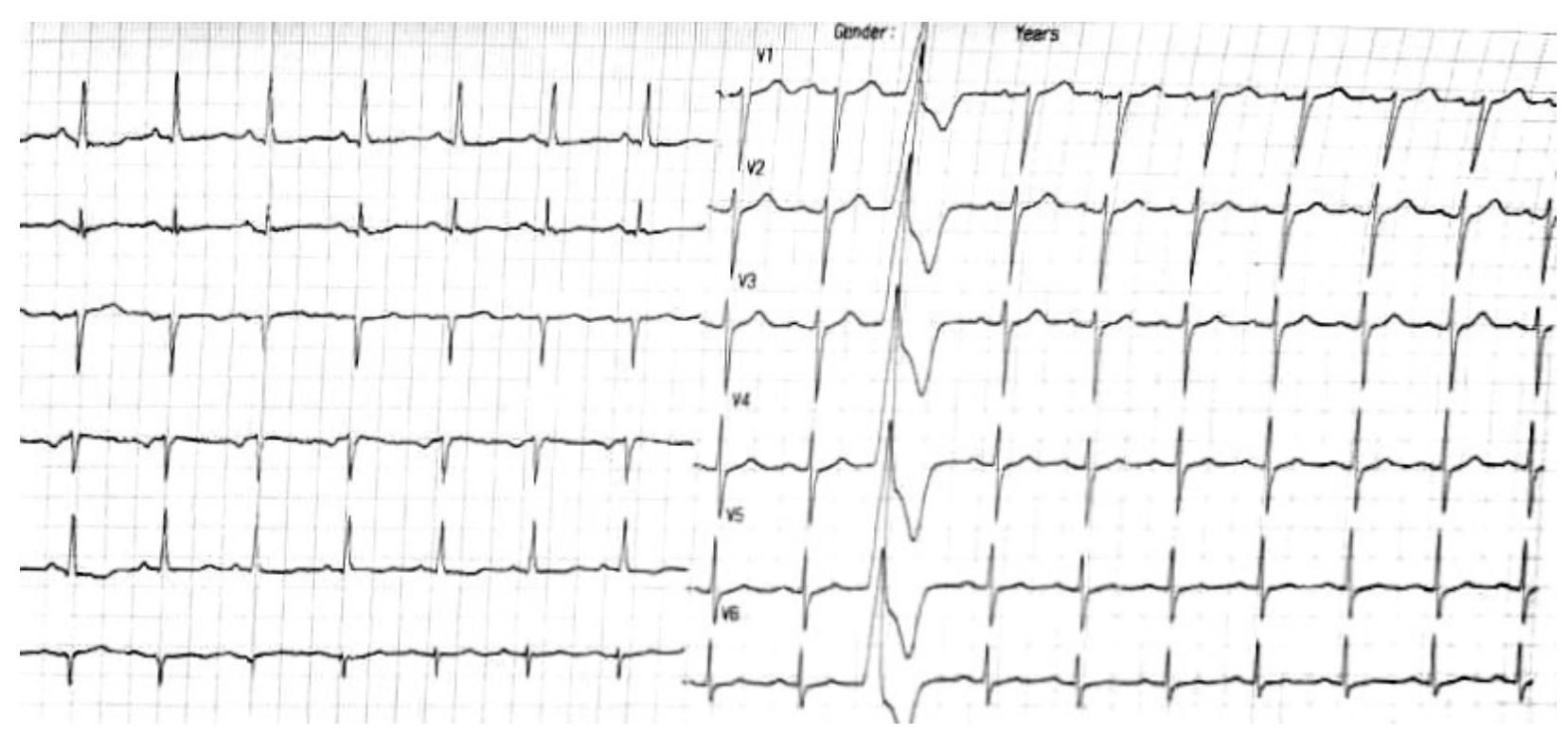

Figure 1: Initial ECG done prior to initiation of hydroxychloroquine and azithromycin. 


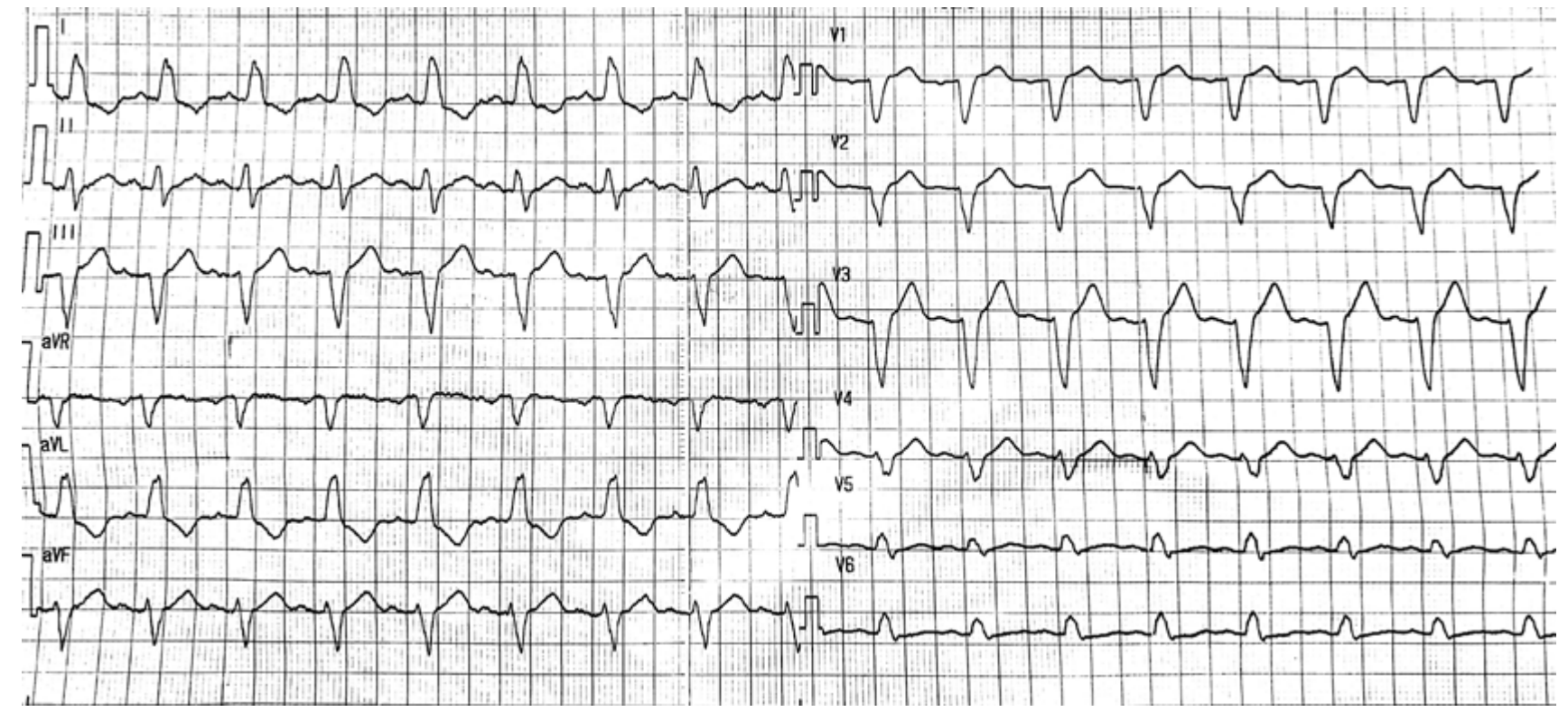

Figure 2: The ECG that was done after second dose of hydroxychloroquine and showed left-bundle branch block.

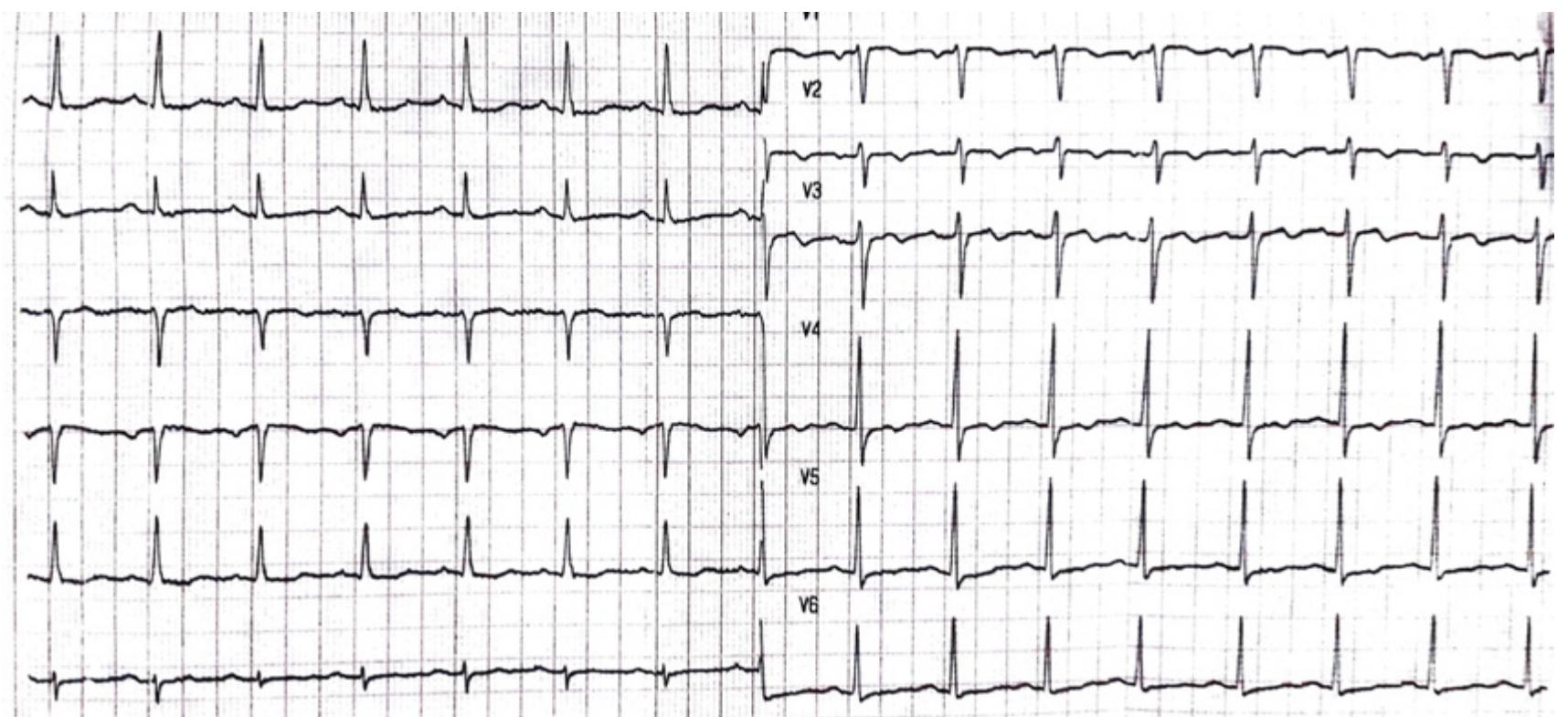

Figure 3: The ECG that was done on Day 6 after stopping the treatment that showed no left-bundle branch block.

The RT-PCR COVID-19 came back positive (cycle threshold: 16.38) and after discussing therapeutic options currently available with the patient, the decision was made to start HCQ 200 mg orally every 8 hours for 10 days in combination with AZT $500 \mathrm{mg}$ orally, once as an initial dose, then to continue $250 \mathrm{mg}$ orally daily for 4 days.
Cardiac clearance was obtained prior to initiation of this regimen as there were no contraindications, no QT prolongation on ECG, no association with other pro-arrhythmic drugs, and no electrolytes or liver function test disturbances. A repeat ECG 2-3 hours after the second dose of HCQ and 12 hours after loading of AZT showed sinus tachycardia and complete LBBB (Figure 2). 
The patient remained completely asymptomatic and was clinically and haemodynamically stable, with no signs of fluid overload, jugular venous distension, or peripheral oedema.

Blood workup was repeated, including cardiac enzymes, D-dimers, and pro-brain natriuretic peptide, and was always negative. Arterial blood gas readings were within normal. The regimen was aborted, and the patient was kept on telemetry and symptomatic treatment. Repeat ECG 24 hours after stopping the treatment showed persistent LBBB. During the 5 days following the cessation of the regimen, his daily ECG showed LBBB, and on the Day 6, his ECG reverted to normal without LBBB (Figure 3). A complete transthoracic echocardiography was performed showed no significant findings.

\section{DISCUSSION}

$\mathrm{HCQ}$ and chloroquine (CQ) have been used to treat malaria infection and connective tissue disorders such as systemic lupus erythematosus. A high incidence of cardiac conduction disorders and atrioventricular block has been observed with $C Q .{ }^{2}$ Severe cardiac toxicities were also observed with acute and chronic use of HCQ, such as TdP, QT prolongation, decreased resting heart rate, and refractory ventricular arrhythmias., ${ }^{3,4}$ On the other hand, the widely used antibiotic AZT is increasingly recognised as a rare cause of $Q T$ prolongation, serious arrhythmias, and increased risk for sudden death, especially in advanced age and the female sex. Interestingly, azithromycin can also provoke non-pause-dependent polymorphic ventricular tachycardia. The U.S. Food and Drug Administration (FDA) perspective supported the observations that AZT administration leaves the patient vulnerable to QTC interval prolongation and TdP.

In December 2019, China reported a cluster of pneumonia cases that were identified as SARSCoV-2. Patients with COVID-19 frequently present with fever, cough and shortness of breath within 2-14 days of exposure. ${ }^{5}$

Direct myocardial injury occurs via the binding of the virus to angiotensin-converting enzyme 2 , a membrane-bound aminopeptidase that is highly expressed in heart, leading to alteration of the angiotensin-converting enzyme 2 signalling pathway. ${ }^{6}$ Increased cardiometabolic demand, associated with systemic infection, coupled with hypoxia caused by acute respiratory illness, can impair the myocardial oxygen demand-supply relationship and additionally lead to acute myocardial injury. ${ }^{7}$

HCQ is a cationic, weak amphiphilic base that crosses cell membranes and binds to phospholipids, accumulates in lysosomes, and causes direct inhibition of phospholipases. ${ }^{8}$ Immunohistochemistry has revealed the accumulation of the autophagic markers, LC3 and p62, suggesting a potential role of autophagy in the pathophysiology of HCQ-induced cardiomyopathy. ${ }^{9}$ The accumulation of these metabolic products leads to the development of cellular hypertrophy of cardiac myocytes and myocardial fibrosis. ${ }^{8}$ Ultimately, these changes result in a cardiomyopathy with concentric hypertrophy and restrictive features, along with conduction abnormalities. ${ }^{8}$

AZT is a semi-synthetic macrolide antibiotic thought to prolong the QT interval through a blockade of the rapid component, $\mathrm{IKr}$, of the delayed rectifier potassium current IK, which is encoded by the human ether-ago-go-related gene 1 ( $h E R G 1$ ), leading to intracellular accumulation of potassium and ventricular repolarisation. ${ }^{10}$

Drug-drug interaction may explain a prolonged QT interval. When AZTs are used with other QTprolonging drugs such as HCQ, they may inhibit CYP enzymes and reduce the metabolism of other drugs by forming an inactive CYP complex."

In an ongoing study on patients included in a single-arm protocol from early March to $16^{\text {th }}$ March, HCQ treatment has been significantly associated with viral load reduction/ disappearance in patients with COVID-19 and its effect is reinforced by $A Z T .^{12}$ Consequently, given their potential risk for arrhythmias and QT prolongation, published data reported the impact of the association of both drugs in treating infected patients, and thereby recommendations to prevent cardiotoxicity. ${ }^{13}$

Transient bundle branch block is defined as an intraventricular conduction defect, that, if only temporarily, subsequently returns to normal conduction. ${ }^{14}$ It has been associated with several conditions such as bradycardia, tachycardia, anaesthesia, acute pulmonary 
embolism, changes in intrathoracic pressure, chest trauma, cardiac interventional procedures, and other clinical conditions. ${ }^{14}$ Apart from coronary artery disease, other causes of LBBB include valvular heart disease, cardiomyopathies (dilated, infiltrative, hypertensive) congenital heart disease, degenerative-conduction heart disease, myocarditis, infective endocarditis, heart trauma/surgery, hyperkalaemia, myxoedema, and systemic sclerosis. ${ }^{14}$

The authors' patient was known to be previously hypertensive, is 58-years old, and had no previously known history of heart failure or myocardial infarction. He was not septic, off diuretics, and had normal QTC on baseline ECG and normal electrolytes. His Tisdale Score was 3. Therefore, he had no contraindications for receiving the regimen.

In a study on 85 unselected outpatients (79 females and 6 males) routinely followed up for 1 year after January 2003, all treated with HCQ for lupus erythematosus, only one patient developed complete LBBB. ${ }^{2}$ The authors' patient had no associated signs or symptoms of myocardial infarction or new onset of heart failure, given his clinical and haemodynamic stability and the normal series of blood biomarkers. His vital signs remained stable without change in heart rate throughout his stay. His ECG findings including left ventricular function, regional wall motions, valves, and pressures were all within normal.
Although acute ECG changes such as T-wave inversion occur in response to administration of $C Q / H C Q$, major conduction abnormalities in the acute setting are much less common than chronic $C Q$ toxicity.

Nevertheless, while all biomarkers and ECG were normal, one cannot exclude a low-level myocarditis, which could be present in the absence of any positive biomarkers. Cardiac MRI was not available for confirmation as myocarditis might have been picked up as a hyperintense signal on T2-weighted images, suggestive of myocardial oedema without significant fibrosis. Hence, one cannot fully exclude COVID-19 myocarditis, which might also show a similar time course. Because of that limitation, one cannot ascribe the ECG changes to the drugs with $100 \%$ certainty.

In conclusion, it is known that the chronic use of $\mathrm{HCQ}$, when previously prescribed for malaria or lupus, is associated with an increase in cardiac toxicity, cardiomyopathies, and arrhythmia, secondary to its cumulative effect, and especially when combined with another cardiotoxic agent. However, new-onset LBBB after only the second dose of $H C Q$ is interestingly debatable, especially when this drug is associated with another arrhythmogenic agent such as AZT.

\section{References}

1. Roden $\mathrm{D}$ et al. Considerations for drug interactions on QTC in exploratory COVID-19 treatment. J Am Coll Cardiol. 2020;75(20):2623-4.

2. Costedoat-Chalumeau $\mathrm{N}$ et al. Heart conduction disorders related to antimalarials toxicity: an analysis of electrocardiograms in 85 patients treated with hydroxychloroquine for connective tissue diseases. Rheumatology (Oxford). 2007;46(5):808-10.

3. Chen $\mathrm{CY}$ et al. Chronic hydroxychloroquine use associated with QT prolongation and refractory ventricular arrhythmia. Clin Toxicol (Phila). 2006;44(2):173-5.

4. Cairoli E et al. Cumulative dose of hydroxychloroquine is associated with a decrease of resting heart rate in patients with systemic lupus erythematosus: a pilot study. Lupus. 2015;24(11):1204-9

5. Dhama K et al. Coronavirus Disease 2019 - COVID-19. Clin Microbiol Rev. 2020; 33(4):e00028-20. DOI:10.20944/preprints202003.0001. v2.

6. Xiong TY et al. Coronaviruses and the cardiovascular system: acute and long-term implications. Eur Heart J. 2020;41(19):1798-800.

7. Li B et al. Prevalence and impact of cardiovascular metabolic diseases on COVID-19 in China. Clin Res Cardiol. 2020;109(5):531-8.

8. Tönnesmann E et al. Chloroquine cardiomyopathy - a review of the literature. Immunopharmacol Immunotoxicol. 2013;35(3):434-42.

9. Daniels $\mathrm{BH}$ et al. LC3 and p62 as diagnostic markers of drug-induced autophagic vacuolar cardiomyopathy: a study of 3 cases. Am J Surg Pathol. 2013;37(7):1014-21.

10. Owens RC Jr, Nolin TD. Antimicrobialassociated QT interval prolongation: pointes of interest. Clin Infect Dis. 2006;43(12):1603-11.

11. Guo D et al. The cardiotoxicity of macrolides: a systematic review. Pharmazie. 2010;65(9):631-40.

12. Gautret P et al. Hydroxychloroquine and azithromycin as a treatment of COVID-19: results of an openlabel non-randomized clinical trial. Int J Antimicrob Agents. 2020;56(1):105949. [Epub ahead of print].

13. Simpson $\mathrm{T}$ et al. Ventricular arrhythmia risk due to hydroxychloroquine-azithromycin 
treatment for COVID-19. March

2020. Available at: https://www

acc.org/latest-in-cardiology/

articles/2020/03/27/14/00/

ventricular-arrhythmia-risk-due-to- hydroxychloroquine-azithromycintreatment-for-covid-19. Last

accessed: 18 August 2021

branch block-a comprehensive

review of the literature. Ann

Noninvasive Electrocardiol.

14. Bazoukis $\mathrm{G}$ et al. Episodic left bundle 